\title{
Exact solution in the discrete case for solitons propagating in a chain of harmonically coupled particles lying in double-minimum potential wells
}

\author{
V. Hugo Schmidt \\ Department of Physics, Montana State University, Bozeman, Montana 59717
}

(Received 19 March 1979)

\begin{abstract}
Solitons of the form $x_{n}=x_{0} \tanh (\omega t-k n a)$ can propagate in a chain of harmonically coupled particles in the discrete case if the potential $-\frac{1}{2} A x_{n}^{2}+\frac{1}{4} B x_{n}^{4}$ giving such solitons in the continuum limit is suitably modified. This modified potential is expressible in closed form, and its shape is a function of $\omega$ and $k$. For large $\omega$ the maximum at $x_{n}=0$ becomes a minimum, giving a triple-minimum potential. Potential shapes and particle positions are illustrated for various $(\omega, k)$ combinations. The total energy and its kinetic, potential, and spring energy constituents are also expressible in closed form. In the continuum limit the total energy has the form $E=m_{0} c_{s}^{2} /\left(1-v^{2} / c_{s}^{2}\right)^{1 / 2}$, where $m_{0}$ is the soliton effective mass, $v$ is the soliton speed, and $c_{s}$ is the speed of sound in the mass-spring chain.
\end{abstract}

\section{INTRODUCTION}

Solitons or solitary waves occur in a wide variety of physical situations. ${ }^{1,2}$ Krumhansl and Schrieffer ${ }^{3}$ studied solitons for particles in a chain of doubleminimum wells having the Hamiltonian

$H=\sum_{n}\left(\frac{p_{n}^{2}}{2 m}-\frac{1}{2} A x_{n}^{2}+\frac{1}{4} B x_{n}^{4}+\frac{1}{2} c\left(x_{n+1}-x_{n}\right)^{2}\right)$,

and showed that in the continuum limit for which displacements of adjacent particles are nearly the same, these displacements are given by

$$
x_{n}=x_{0} \tanh (\omega t-k n a)
$$

Here $a$ is the distance between wells, $n$ is the well index, and $x_{0}$ is the distance $(A / B)^{1 / 2}$ from the potential maximum at the center of the well to either minimum. The quantity $\omega / k$ is the soliton velocity, which of course is different from the instantaneous velocity $\omega x_{0} \operatorname{sech}^{2}(\omega t-k n a)$ of the $n$th particle. Recently, Mazenko and Sahni ${ }^{4}$ have extended the statistical treatment of this continuum model, including the effect of phonons.

A quantum-mechanical treatment by Bishop, Domany, and Krumhansl ${ }^{5}$ predicted qualitatively similar behavior to that found for the above classical model.

A number of computer simulation studies have been made for the above $-\frac{1}{2} A x_{n}^{2}+\frac{1}{4} B x_{n}^{4}$ potential system in the discrete region away from the continuum limit. ${ }^{6-8}$ Similar studies have been done ${ }^{9-11}$ for systems obeying the sine-Gordon equation, for which the potential is sinusoidal and a given particle can migrate from minimum to minimum, rather than being confined to its own double-minimum well. Soliton energy loss to phonons was found for the discrete case, but such soliton-phonon interactions were absent in the continuum limit. ${ }^{9}$

The sine-Gordon solitons are applicable to conduction problems such as charge-density waves and Josephson-junction transmission lines. The $-\frac{1}{2} A x_{n}^{2}+\frac{1}{4} B x_{n}^{4}$ double-mimimum potential system is applicable to structural phase transitions of some types, such as certain ferroelectric transitions, and to the ac dielectric response of coupled charged particles in a chain of double-minimum cells. In many of these problems the width of the soliton or domain wall is only a few lattice spaces, so that discreteness effects become important. It would be useful to have exact solutions for the discrete case in addition to the computer simulation results. A general approach to such exact solutions has been given by Ablowitz and Ladik, ${ }^{12,13}$ but they provided no examples of application of their formalism to simple potentials or simple soliton waveforms.

The present work was motivated by a desire to better understand the dynamics of cluster growth and decay near phase transitions, and of domain-wall mobility, in hydrogen-bonded ferroelectrics in which the double-minimum hydrogen bond plays a central role. ${ }^{14}$ In such crystals the domain wall is only a few lattice constants wide, ${ }^{15}$ corresponding to a soliton for which the continuum limit is a poor approximation.

To gain understanding of motions of such domain walls or cluster boundaries, we investigate here a related one-dimensional soliton model. At first we tried the expression

$$
x_{n}=\sum_{j=0}^{\infty} x_{j} \tanh ^{2 j+1}(\omega t-k n a),
$$

for the displacements of coupled particles lying in a chain of double-minimum potential wells of the form 
$U_{n}\left(x_{n}\right)=-\frac{1}{2} A x_{n}^{2}+\frac{1}{4} B x_{n}^{4}$. After difficulties were encountered in finding the coefficients $x_{j}$, we followed a suggestion by Kinnersley ${ }^{16}$ that only the first term $x_{0} \tanh (\omega t-k n a)$ be used for the displacement and that the potential be found which yields this displacement. We expected an infinite power series in $x_{n}^{2}$ for this potential, but it turned out to be expressible in closed form. This paper presents the derivation of this potential, and illustrates various potential shapes and the corresponding particle displacements for stationary and propagating solitons. The kinetic, potential, and spring energy contributions to the soliton energy are also calculated. A subsequent paper will discuss the extent to which results from this onedimensional model are directly applicable to soliton motion in certain two- and three-dimensional models, including a three-dimensional model which is quite a good representation of ferroelectric crystals having the $\mathrm{KH}_{2} \mathrm{PO}_{4}$-type structure.

\section{MODEL AND EQUATION OF MOTION}

The model can be visualized as shown in Fig. 1, in which the masses $m$ move only in the horizontal direction and are coupled by springs of spring constant $c$. The wells are spaced at intervals $a$ and the $n$th mass has displacement $x_{n}$ from the center of the well. The potential minima are located at $\pm x_{0}$ relative to the well center. A soliton appears in Fig. 1, because the wells on the left have particles in the right-hand minima, while the wells on the right have particles in the left-hand minima. The compressive spring forces prevent the particles shown from being located exactly at the potential minima. The stronger the springs, the greater the width of the soliton will be, and the closer it will come to satisfying the continuum approximation.

The form $x_{n}=x_{0} \tanh (\omega t-k n a)$ chosen for the particle displacement corresponds to a compressive soliton as shown in Fig. 1 if $k$ is positive. The soliton travels to the right for positive $\omega$, and to the left for negative $\omega$. Negative $k$ corresponds to a dilative soliton as shown in Fig. 2, which travels to the right for negative $\omega$, and to the left for positive $\omega$. We derive

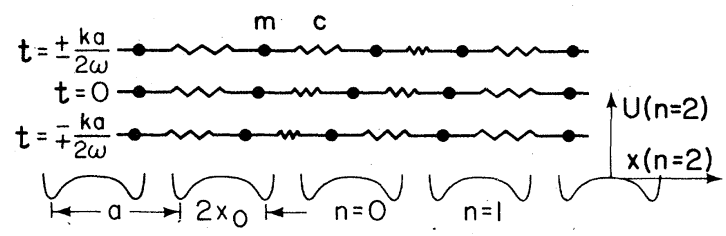

FIG. 1. Compressive soliton having parameters $\tau=\Omega=0.8$ shown at the beginning, middle, and end of one cycle of motion. It is traveling to the right for times indicated by the upper signs, and to the left for the lower signs.

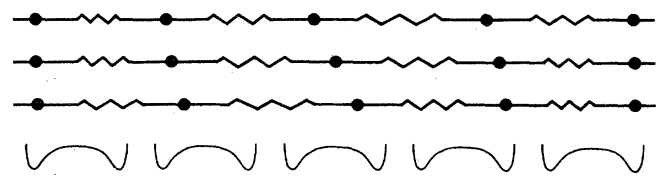

FIG. 2. Dilative soliton having parameters $\tau=\Omega=0.8$ shown at the beginning, middle, and end of one cycle of motion. The legend of Fig. 1 applies. Note that as the soliton moves to the right, the individual particles are moving leftward.

here the potential $U_{n}\left(x_{n}\right)$ which yields the above form for the particle displacement. We will find that this potential depends on the magnitudes but not on the signs of $k$ and $\omega$.

The displacement expression $x_{n}=x_{0} \tanh (\omega t$ $-k n a$ ) corresponds to a soliton which propagates by means of each particle moving in turn a distance $2 x_{0}$ from one side of its well to the other. To find the potential which yields this type of motion, the equation of motion is written with the potential in arbitrary power-series form. The power-series coefficients are then found which yield particle displacements obeying Eq. (2). Finally, the potential is expressed in closed form.

The equation of motion for the $n$th particle is

$\frac{m d^{2} x_{n}}{d t^{2}}=\frac{-d U_{n}}{d x_{n}}+c\left(x_{n+1}+x_{n-1}-2 x_{n}\right)$,

where the potential $U_{n}$ is given by

$U_{n}=-\frac{1}{2} A x_{n}^{2}+\frac{1}{4} B x_{n}^{4}+\frac{1}{6} C x_{n}^{6}+\frac{1}{8} D x_{n}^{8}+\cdots$.

We now define $T_{n} \equiv \tanh (\omega t-k n a)$ and $\tau \equiv \tanh (k a)$ and obtain

$$
\begin{aligned}
& \frac{d^{2} x_{n}}{d t^{2}}=2 \omega^{2} x_{0} T_{n}\left(T_{n}^{2}-1\right), \\
& x_{n+1}+x_{n-1}=2 x_{0}\left(1-\tau^{2}\right) T_{n}\left(1+\tau^{2} T_{n}^{2}+\tau^{4} T_{n}^{4}+\cdots\right),
\end{aligned}
$$

and

$\frac{d U_{n}}{d x_{n}}=-A x_{n}+B x_{n}^{3}+C x_{n}^{5}+D x_{n}^{7}+\cdots$.

From here on the subscript $n$ can be dropped without confusion.

We now convert to a dimensionless position variable $y \equiv x / x_{0}$, a dimensionless potential $V \equiv U / c x_{0}^{2}$, and a dimensionless parameter $\Omega^{2} \equiv \omega^{2} m / c$ related to the soliton velocity. With these substitutions, the equation of motion upon dividing through by $x_{0} c T$ becomes

$$
\begin{aligned}
& 2 \Omega^{2}\left(T^{2}-1\right)-2\left(1-\tau^{2}\right)\left(1+\tau^{2} T^{2}+\tau^{4} T^{4}+\cdots\right)+2 \\
& \quad+\left(-A+B x_{0}^{2} T^{2}+C x_{0}^{4} T^{4}+D x_{0}^{6} T^{6}+\cdots\right) / c=0 .
\end{aligned}
$$


This equation can be written as a power series in $T^{2}$,

$$
\begin{aligned}
& \left(\tau^{2}-\Omega^{2}-A / 2 c\right)+\left(\Omega^{2}+B x_{0}^{2} / 2 c-\tau^{2}+\tau^{4}\right) T^{2} \\
& +\left(C x_{0}^{4} / 2 c-\tau^{4}+\tau^{6}\right) T^{4} \\
& \quad+\left(D x_{0}^{6} / 2 c-\tau^{6}+\tau^{8}\right) T^{6}+\cdots=0 .
\end{aligned}
$$

Because $T$ is a function of time $t$ and well index $n$, Eq. (10) is satisfied for all $t$ and $n$ only if each coefficient in parentheses vanishes. Accordingly,

$$
\begin{aligned}
& A=2 c\left(\tau^{2}-\Omega^{2}\right), \\
& B=2 c\left(\tau^{2}-\tau^{4}-\Omega^{2}\right) / x_{0}^{4}, \\
& C=2 c\left(\tau^{4}-\tau^{6}\right) / x_{0}^{4}, \\
& D=2 c\left(\tau^{6}-\tau^{8}\right) / x_{0}^{6}, \text { etc. }
\end{aligned}
$$

The dimensionless potential $V$ in terms of the dimensionless position $y$ then becomes

$$
\begin{aligned}
V(y)= & \left(-\frac{1}{2} A x_{0}^{2} y^{2}+\frac{1}{4} B x_{0}^{4} y^{4}+\frac{1}{6} C x_{0}^{6} y^{6}\right. \\
& \left.+\frac{1}{8} D x_{0}^{8} y^{8}+\cdots\right) / c x_{0}^{2} \\
= & \left(\Omega^{2}-\tau^{2}\right) y^{2}+\frac{1}{2}\left(\tau^{2}-\tau^{4}-\Omega^{2}\right) y^{4} \\
& +\frac{1}{3}\left(\tau^{4}-\tau^{6}\right) y^{6}+\frac{1}{4}\left(\tau^{6}-\tau^{8}\right) y^{8}+\cdots \\
= & \Omega^{2} y^{2}-\frac{1}{2} \Omega^{2} y^{4}-y^{2} \\
& -\left(1-\tau^{-2}\right)\left(\tau^{2} y^{2}+\frac{1}{2} \tau^{4} y^{4}+\frac{1}{3} \tau^{6} y^{6}+\frac{1}{4} \tau^{8} y^{8}+\cdots\right) \\
= & \left(\Omega^{2}-1\right) y^{2}-\frac{1}{2} \Omega^{2} y^{4} \\
& +\left(1-\tau^{-2}\right) \ln \left(1-\tau^{2} y^{2}\right)
\end{aligned}
$$

This potential remains finite if $\tau$ and $y$ are restricted to physically meaningful values, namely $\tau^{2}<1$ so that $k$ and the soliton and particle propagation velocities remain finite, and $y^{2} \leqslant 1$ because from Eq. (2) one sees that $y^{2} \equiv x^{2} / x_{0}^{2}$ is restricted to that range.

\section{RESULTS FROM MODEL}

Plots of the dimensionless potential $V(y)$ for various values of $\tau \equiv \tanh (k a)$ and $\Omega \equiv \omega^{2} \mathrm{~m} / c$ appear in Figs. 3 and 4 . Circles indicate particle positions at the instant that a particle is at the center of the well (at $y=0)$. Each successive circle as one goes to the right along a potential curve corresponds to the particle position in the next well to the right for a dilative soliton as shown in Fig. 2, or to the particle position in the next well to the left for a compressive soliton as shown in Fig. 1. The particle positions shown in Figs. 3 and 4 correspond to $y=\tanh (k n a)$ which is the $t=0$ form of the general expression for $y$, with $n=0,1,2, \ldots$ for dilative solitons and $n=0,-1$, $-2, \ldots$ for compressive solitons. Note that $y=\tau$

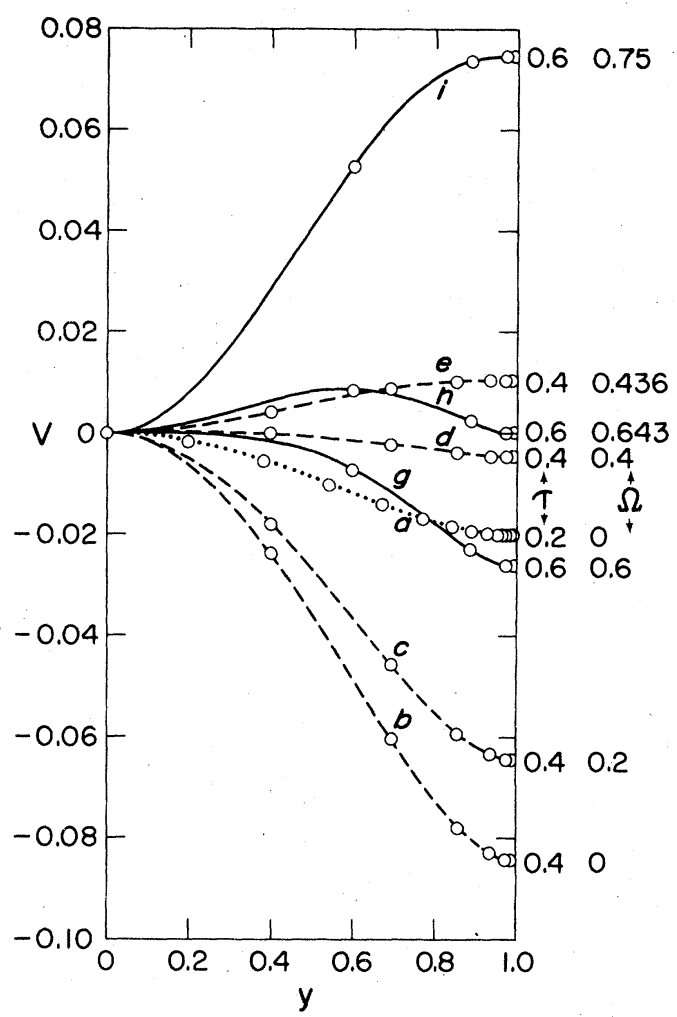

FIG. 3. Shape of the right half of the dimensionless potential $V(y)$ for various values of the parameters $\tau$ and $\Omega$. Dotted curve is for $\tau=0.2$, dashed curves for $\tau=0.4$ and solid curves for $\tau=0.6$. Adjacent circles on each curve correspond to positions of particles in adjacent wells, at the instant that a particle is at the center of the well $(y=0)$. Curves are labeled by letters for reference to points in Fig. 5. Curve $g$ appears also in Fig. 4, on a different scale.

for $|n|=1$. The particle positions crowd together as $y$ approaches 1 , corresponding to particles almost undisturbed by the approaching or receding soliton.

The potentials for $\Omega=0$ corresponding to stationary solitons have only two minima, at $y= \pm 1$. With increasing $\Omega$ for given $\tau$ the potential curves lie higher, until for $\Omega=\tau$ a third minimum appears at $y=0$. For a certain larger $\Omega$ the three minima all lie at $V=0$, while for some still larger value of $\Omega$ the outer minima disappear.

In the limit $\Omega<<\tau<1, V(y)$ approaches the const $\times\left(-\frac{1}{2} y^{2}+\frac{1}{4} y^{4}\right)$ shape known previously ${ }^{3}$ to correspond in the continuum limit to solitons of the form $y=\tanh (k n a)$. As $\tau$ increases the soliton departs from the continuum form and the minima at $y= \pm 1$ become sharper, becoming abrupt in the limit $\tau=1$. In this limit the soliton consists of a single particle moving from one minimum to another in a given well. Only when the particle reaches the second minimum does the particle in the adjacent 


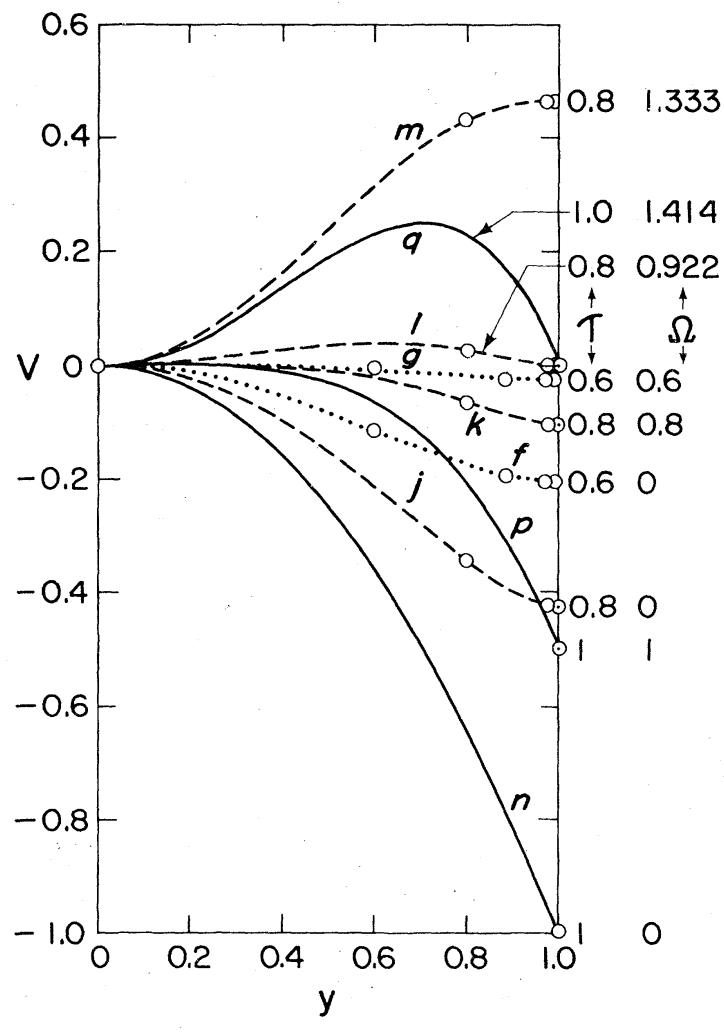

FIG. 4. Caption for Fig. 3 applies, except that dotted curve is for $\tau=0.6$, dashed curves are for $\tau=0.8$ and solid curves are for $\tau=1$.

well begin to move. For the stationary soliton of this type $(\tau=1, \Omega=0)$ shown on curve $n$ of Fig. 4 , the soliton energy is particularly easy to evaluate. The particle shown at $y=0$ at the well center has potential energy relative to the well minima of

$$
E_{p}=U(0)-U\left(x_{0}\right)=c x_{0}^{2}[V(0)-V(1)]=c x_{0}^{2} .
$$

Each of the springs joined to this particle is stretched an amount $x_{0}$, so the total spring energy is also $c x_{0}^{2}$, giving total energy $2 c x_{0}^{2}$ for the soliton. If this particle is now moved to one of the minima of its well, no other particle will move. One spring will then be relaxed, while the other will be stretched an amount $2 x_{0}$, so the spring energy will now be $2 c x_{0}^{2}$ which will still be the total energy. This change $c x_{0}^{2}$ in spring energy is also the maximum potential barrier height for which solitons of the $x_{n}=x_{0} \tanh (\omega t-k n a)$ form can occur.

For this analysis it is convenient to use the massspring system properties as references to establish dimensionless parameters for the solitons and potential wells. For instance, a logical energy reference is the above spring energy difference of $c x_{0}^{2}$, which is used in the definition $V \equiv U / c x_{0}^{2}$. Similarly, it is con-

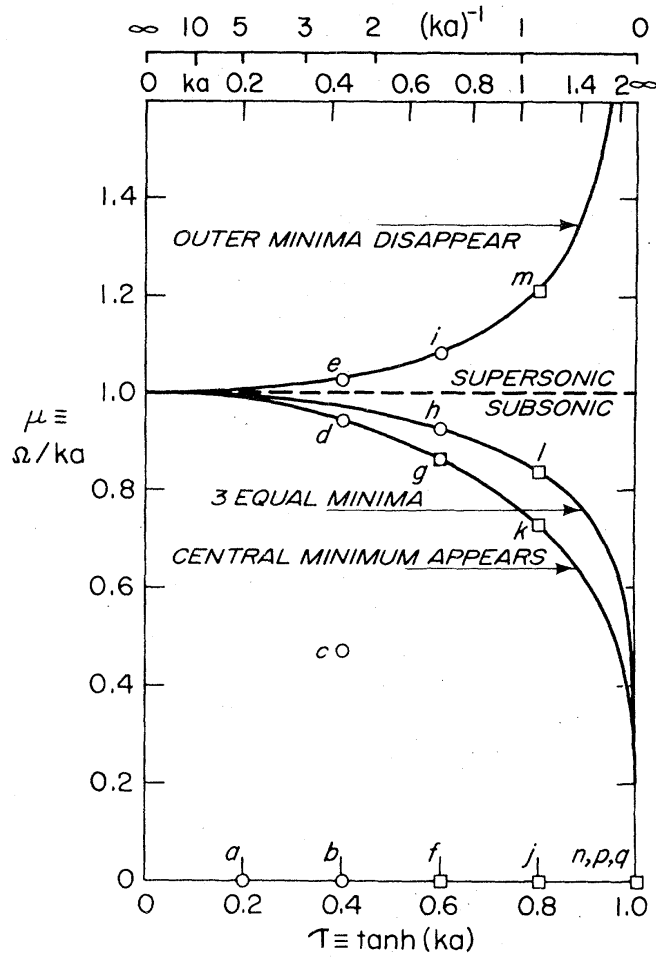

FIG. 5. Plot of the velocity ratio $\mu \equiv v / c_{s}$ as a function of $\tau$ for solitons with the $(\tau, \Omega)$ combinations shown in Fig. 3 (circles) and Fig. 4 (squares). Also shown are the $\mu(\tau)$ curves corresponding to appearance of a central minimum, existence of three equal minima, and disappearance of the outer minima. Scales of $k a$ and of the "soliton half-width" $(k a)^{-1}$ appear at the top.

venient to express the soliton velocity in terms of the phonon velocity for the mass-spring system. If the restoring forces caused by the wells are ignored and only the mass-spring system is considered, the phonon velocity in the long-wavelength limit is $a(c / m)^{1 / 2}$. The soliton velocity is $\omega / k$, so the ratio $\mu$ of the soliton velocity to the phonon velocity is given by $\mu=\Omega / k a$. This ratio is indicated in Fig. 5 for the various $(\tau, \Omega)$ combinations corresponding to the potentials shown in Figs. 3 and 4. Each circle (square) in Fig. 5 corresponds to the curve indicated by the same letter in Fig. 3 (Fig. 4).

The fastest solitons occur for the case of particles moving between two outer minima which are on the verge of disappearing; for this unphysical case the solitons are "supersonic." For potentials with three equal minima, or for which the central minimum just appears, solitons of our chosen hyperbolic tangent form are "subsonic." Note that in the continuum limit $(\tau=0)$ the soliton velocity cannot exceed the phonon velocity. The points $a, b, f, j$, and $n$ correspond to stationary solitons for which the velocity ratio $\mu$ is zero. This ratio is also zero for points $p$ and $q$. For 
these potentials (shown in Fig. 4) the particle leaves and approaches the minima with zero acceleration as well as zero velocity, so that the transit time across the well is infinite even though the velocity at intermediate points in the well is nonzero. This infinite transit time, together with the fact that only one particle moves at a time for such $\tau=1$ solitons, makes the mean soliton velocity zero for these nonstationary solitons.

Near the continuum limit the potential can be expanded as

$$
\begin{aligned}
V(y)= & {\left[\Omega^{2}-k^{2} a^{2}\left(1-\frac{2}{3} k^{2} a^{2}+\cdots\right)\right] y^{2} } \\
& +\left[-\frac{1}{2} \Omega^{2}+\frac{1}{2} k^{2} a^{2}\left(1-\frac{5}{3} k^{2} a^{2}+\cdots\right)\right] y^{4} \\
& +\frac{1}{3} k^{4} a^{4}\left(1-\frac{7}{3} k^{2} a^{2}+\cdots\right) y^{6}+\cdots,
\end{aligned}
$$

from which the dimensionless barrier height is

$\Delta V \equiv V(0)-V(1)=\frac{1}{2}\left(k^{2} a^{2}-\Omega^{2}\right)-\frac{1}{6} k^{4} a^{4}+\cdots$.

In the continuum limit $V(y)=-\frac{1}{2} A^{\prime} y^{2}+\frac{1}{4} B^{\prime} y^{4}$, where $A^{\prime}=B^{\prime}=2\left(k^{2} a^{2}-\Omega^{2}\right)$, and $\Delta V=\frac{1}{4} A^{\prime}$.

In the short-soliton limit $(k a \rightarrow \infty)$ the potential becomes

$$
\begin{aligned}
V(y)= & \left(\Omega^{2}-1\right) y^{2}-\frac{1}{2} \Omega^{2} y^{4}-\left(4 e^{-2 k a}+\cdots\right) \\
& \times \ln \left[1-\left(1-4 e^{-2 k a}+\cdots\right) y^{2}\right] .
\end{aligned}
$$

As the limit is approached and the last term vanishes, the potential has only a quadratic and quartic term as in the continuum limit. However, instead of approaching a minimum smoothly as in the continuum limit, $V(y)$ approaches the $y=1$ minimum with slope -2 , independent of $\Omega$, as seen in Fig. 4 . In this limit the barrier height is given by $\Delta V=1-\frac{1}{2} \Omega^{2}$.

The above barrier heights are limiting cases of the general expression

$$
V(0)-V(1)=1-\frac{1}{2} \Omega^{2}+\left(\tau^{-2}-1\right) \ln \left(1-\tau^{2}\right) .
$$

This definition for $\Delta V$ is meaningful only for $\Omega \leqslant \tau$. For $\Omega>\tau$ the maximum at $y=0$ becomes a minimum, and maxima appear at $Y= \pm\left(\tau^{-2}-\Omega^{-2}\right)^{1 / 2}$ having the value

$$
\begin{aligned}
V_{\max }= & \Omega^{2} \tau^{-2}-1+\frac{1}{2} \Omega^{-2}-\frac{1}{2} \Omega^{2} \tau^{-4} \\
& +\left(\tau^{-2}-1\right) \ln \left(\Omega^{2} \tau^{-2}\right)
\end{aligned}
$$

As $\Omega$ is increased to $\left[2-2\left(\tau^{-2}-1\right) \ln \left(1-\tau^{2}\right)\right]^{1 / 2}$ the minima at $y= \pm 1$ rise to the value $V=0$ possessed by the $y=0$ minimum. For still larger $\Omega$ the outer minima are metastable. When $\Omega$ increases to $\left(\tau^{-2}-1\right)^{-1 / 2}$ the maxima move out to $y= \pm 1$ and the metastable minima disappear.
The total soliton energy is constant because the model provides no energy gain or loss mechanisms. However, the partition of this energy among kinetic energy, potential energy of position in the wells, and interaction or spring energy will vary over a cycle of motion during which the soliton moves one lattice space. Particle positions at the beginning, middle, and end of one cycle are illustrated in Figs. 1 and 2. Calculation of each of these energy contributions for a given point in the cycle requires summation of the contributions from each particle or spring. Fortunately the time averages of the kinetic, potential, and spring energies can be expressed in integral form. For example, the time average of the kinetic energy $E_{k}$ can be expressed as

$$
\begin{aligned}
\left\langle E_{k}\right\rangle & =\left(\frac{\omega}{k a}\right) \int_{0}^{k a / \omega} d t \sum_{n=-\infty}^{\infty} E_{k n}(\omega t-k n a) \\
& =2 \int_{0}^{\infty} d n E_{k n}(-k n a) .
\end{aligned}
$$

Because the kinetic energy $E_{k n}$ of the $n$th particle at $t=0$ can be expressed simply in terms of the value $y=\tanh (-k n a)$ which $y$ assumes at $t=0$, Eq. (21) can be expressed as

$$
\begin{aligned}
\left\langle E_{k}\right\rangle & =2 \int_{0}^{-1} d y\left(\frac{d y}{d n}\right)^{-1} E_{k n}(y) \\
& =\left(\frac{2}{k a}\right) \int_{0}^{1} d y\left(1-y^{2}\right) E_{k n}(y) .
\end{aligned}
$$

Substitution of $E_{k n}(y)=\frac{1}{2} m \omega^{2} x_{0}^{2}\left(1-y^{2}\right)^{2}$ into Eq. (22) yields

$$
\left\langle E_{k}\right\rangle=\frac{\frac{2}{3} m x_{0}^{2} \omega^{2}}{k a}=\frac{\frac{2}{3} c x_{0}^{2} \Omega^{2}}{k a}=\frac{2}{3} k a \mu^{2} c x_{0}^{2} .
$$

The soliton momentum can be found by substituting $p_{n}(y)=m \omega x_{0}\left(1-y^{2}\right)$ for $E_{k n}(y)$ in Eq. (22)

$$
\langle p\rangle=\frac{2 m \omega x_{0}}{k a}=\frac{2(m c)^{1 / 2} \Omega x_{0}}{k a}=2(m c)^{1 / 2} x_{0} \mu .
$$

The momentum is not constant over a displacement cycle because the potential wells exert forces on the particles. Both $\langle p\rangle$ and $\left\langle E_{k}\right\rangle$ are the same as $p$ and $E_{k}$ found in the continuum limit.

The potential energy $E_{p n}$ of the $n$th particle relative to its energy at the $y= \pm 1$ minima is

$$
\begin{gathered}
E_{p n}=c x_{o}^{2}\left[\left(\Omega^{2}-1\right)\left(y^{2}-1\right)-\frac{1}{2} \Omega^{2}\left(y^{4}-1\right)\right. \\
\left.-\left(\tau^{-2}-1\right) \ln \left(\frac{1-\tau^{2} y^{2}}{1-\tau^{2}}\right)\right],
\end{gathered}
$$

obtained by adding the expressions in Eqs. (15) and (19) and multiplying by $c x_{0}^{2}$. Substitution of $E_{p n}$ for 
$E_{k n}$ in Eq. (22) yields (see Appendix)

$$
\left\langle E_{p}\right\rangle=\left(\frac{2 c x_{0}^{2}}{k a}\right)\left[1-\frac{1}{3} \Omega^{2}-\left(\tau^{-2}-1\right) k^{2} a^{2}\right] \text {. }
$$

Note that when Eqs. (23) and (26) are added, the terms in $\Omega^{2}$ cancel, so $\left\langle E_{p}\right\rangle+\left\langle E_{k}\right\rangle$ depends only on $k$ and not on $\omega$.

In evaluating the time-averaged spring energy $\left\langle E_{s}\right\rangle$ it is necessary to express the individual spring energy symmetrically when changing the lower integration limit from $-\infty$ to 0 ,

$$
\begin{aligned}
\left\langle E_{s}\right\rangle & =c x_{0}^{2} \int_{0}^{\infty} d n\left\{\tanh \left[k\left(n+\frac{1}{2}\right) a\right]-\tanh \left[k\left(n-\frac{1}{2}\right) a\right]\right\}^{2} \\
& =\left(\frac{4 c x_{0}^{2}}{k a}\right) \tanh ^{2}\left(\frac{1}{2} k a\right) \int_{0}^{1} d y\left(1-y^{2}\right) /\left[1-\tanh ^{2}\left(\frac{1}{2} k a\right) y^{2}\right]^{2}=2 c x_{0}^{2}\left(\frac{1}{\tau}-\frac{1}{k a}\right) .
\end{aligned}
$$

The three energy contributions can now be summed to obtain the total soliton energy $E$

$$
E=\left\langle E_{p}\right\rangle+\left\langle E_{k}\right\rangle+\left\langle E_{s}\right\rangle=2 c x_{0}^{2}\left(\tau^{-1}-k a \tau^{-2}+k a\right) \text {. }
$$

Plots of $E$, of $\left\langle E_{p}+E_{k}\right\rangle$, and of barrier height $\Delta U$ for $\boldsymbol{\Omega}=0$ are shown in Fig. 6 as functions of $\tanh (k a)$

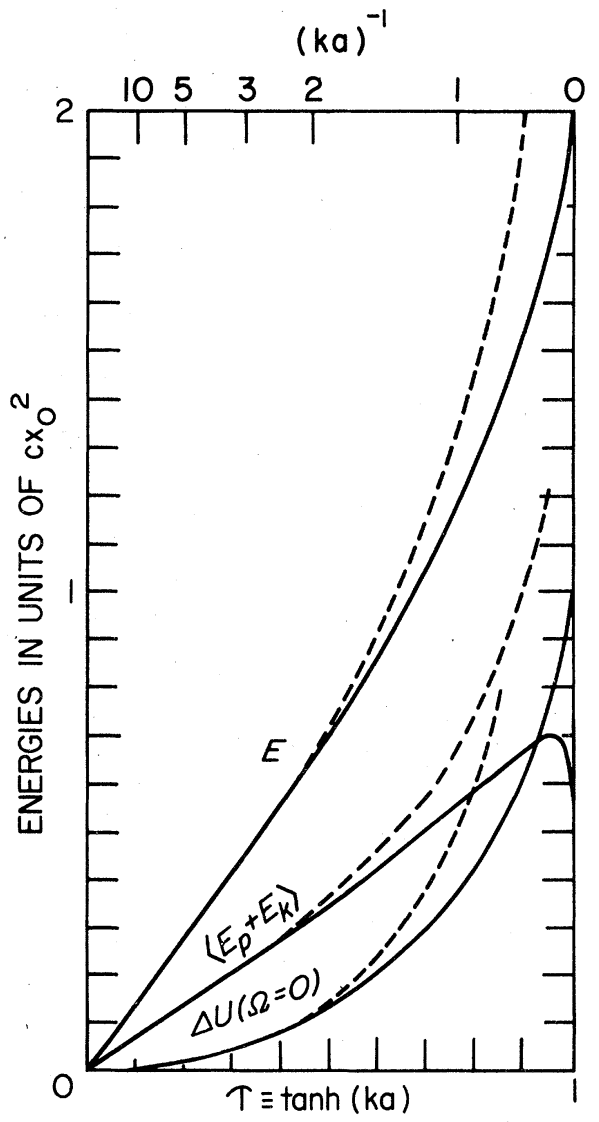

FIG. 6. Plots of the exact (solid lines) and continuum limit (dashed lines) expression for total soliton energy $E$, time average of sum of potential energy of position in well and kinetic energy $\left\langle E_{p}+E_{k}\right\rangle$, and of barrier height $\Delta U$ for stationary ( $\Omega=0$ ) solitons, as functions of $\tau$ (bottom scale) and "soliton half-width" $(\mathrm{ka})^{-1}$ (top scale). and of the "soliton half-width" $(k a)^{-1}$ for both exact and continuum-limit expressions. The exact expressions are from Eqs. (19), (23), (26), and (28). The continuum-limit expressions in units of $c x_{0}^{2}$ are $\frac{1}{2} k^{2} a^{2}$ for $\Delta U$ from Eq. (17), $\frac{4}{3} k a$ for $E$ from Eq. (29), and $\frac{2}{3} k a$ for $\left\langle E_{p}+E_{k}\right\rangle$ from Eq. (33).

The total soliton energy $E$ increases monotonically with $\tau$ from 0 in the continuum limit $(\tau=0)$ to $2 c x_{0}^{2}$ in the short-soliton limit $(\tau=1)$. Near the continuum limit,

$$
E=\frac{4}{3} k a\left(1-\frac{2}{15} k^{2} a^{2}+\cdots\right) c x_{0}^{2},
$$

whose limiting value $\frac{4}{3} k a c x_{0}^{2}$ is shown in Fig. 6 as a dashed line lying above the exact expression curve. In the short-soliton limit,

$$
E=2 c x_{0}^{2}\left[1-(4 k a-2) e^{-2 k a}+\cdots\right] .
$$

The time-averaged spring energy $\left\langle E_{s}\right\rangle$ represented in Fig. 6 as the difference between the $E$ and $\left\langle E_{p}+E_{k}\right\rangle$ curves can be expanded near the continuum limit as

$$
\left\langle E_{s}\right\rangle=\frac{2}{3} k a\left(1-\frac{1}{15} k^{2} a^{2}+\cdots\right) c x_{0}^{2} .
$$

Its limiting value $\frac{2}{3} k a c x_{0}^{2}$ is represented by the middle dashed line in Fig. 6 because it is the same as the continuum limit for $\left\langle E_{p}+E_{k}\right\rangle$. In the short-soliton limit,

$$
\left\langle E_{s}\right\rangle=2 c x_{0}^{2}\left(1+2 e^{-2 k a}+\cdots-1 / k a\right) .
$$

The sum of $\left\langle E_{k}\right\rangle$ and $\left\langle E_{p}\right\rangle$ given in Eqs. (23) and (26), respectively, has near the continuum limit the expansion

$$
\left\langle E_{p}+E_{k}\right\rangle=\frac{2}{3} k a\left(1-\frac{1}{5} k^{2} a^{2}+\cdots\right) c x_{0}^{2},
$$

which is slightly smaller than the corresponding $\left\langle E_{s}\right\rangle$ expression in Eq. (31) because of the second term. As $\tau$ increases toward $1,\left\langle E_{p}+E_{k}\right\rangle$ falls farther and farther below $\left\langle E_{s}\right\rangle$, and in fact drops precipitously to zero at the $\tau=1$ limit, while $\left\langle E_{s}\right\rangle$ climbs just as sharply to $2 c x_{0}^{2}$. The climb in $\left\langle E_{s}\right\rangle$ results from the sudden vanishing of the last two terms in Eq. (32) in 
the $\tau=1$ limit, while the drop in $\left\langle E_{p}+E_{k}\right\rangle$ follows from the sudden vanishing of both terms in that limit in the following short-soliton-limit expression:

$$
\left\langle E_{p}+E_{k}\right\rangle=2 c x_{0}^{2}\left[(k a)^{-1}-\left(4 e^{-2 k a}+\cdots\right) k a\right] .
$$

Finally, the exact and continuum-limit curves for $\Delta U$ for $\Omega=0$ are derived from Eqs. (19) and (17), respectively, keeping only the $\frac{1}{2} k^{2} a^{2}$ term in Eq. (17), and multiplying both expressions by $c x_{0}^{2}$. The reason that $E$ and its three constituent parts rise linearly with $\tau$ for small $\tau$, while $\Delta U$ rises quadratically, lies in the fact that the energy per particle varies as $\Delta U$ but the number of particles in the soliton is roughly $2(k a)^{-1}$ which becomes infinite as $\tau$ approaches zero.

The above energies were expressed in terms of $\tau$ and $\Omega$, and it turned out that $E,\left\langle E_{s}\right\rangle$, and $\left\langle E_{p}+E_{k}\right\rangle$ were independent of $\Omega$, that is, of the soliton velocity. From a physical standpoint it is more meaningful to ask how the energies depend on barrier height $\Delta U \equiv c x_{0}^{2} \Delta V$ and on soliton velocity $v \equiv \mu c_{s} \equiv \mu a$ $\times(\mathrm{c} / \mathrm{m})^{1 / 2}$. Such expressions would be complicated in the discrete case but are much simpler in the continuum limit. By eliminating $k a$ from Eq. (23) for $\left\langle E_{k}\right\rangle$ and from $E_{\text {cont }}=\frac{4}{3} k a c x_{0}^{2}$, one obtains,

$$
E_{\text {cont }}=\frac{\frac{4}{3}(2 \Delta V)^{1 / 2} c x_{0}^{2}}{\left(1-v^{2} / c_{s}^{2}\right)^{1 / 2}}
$$

where the soliton "rest mass" is

$$
m_{0}=\frac{4}{3}\left(x_{0} / a\right)^{2}(2 \Delta V)^{1 / 2} m .
$$

These expressions are reminiscent of special relativity, but of course $c_{s}$ is the speed of long-wavelength phonons and not of light! The kinetic energy in the continuum limit then is $\frac{1}{2} m_{0} v^{2}$ for low soliton velocities far from the "relativistic limit."

The foregoing energy and effective-mass expressions agree with the low-soliton-velocity, continuumlimit expressions in Eqs. (41)-(43) of Krumhansl and Schrieffer, ${ }^{3}$ if the exact coefficients in their Ref. 15 are used. (Their $E_{D P}$ corresponds to our $E_{s}+E_{p}$.)

As $\tau$ increases and soliton length decreases, a larger and larger fraction of the energy transfers back and forth among kinetic, potential, and spring energy during the course of a cycle. In Fig. 6 one sees that for $\tau$ near $1,\left\langle E_{p}\right\rangle$ is smaller than $\Delta U$ for a stationary ( $\Omega=0$ ) soliton, even though $E_{p}$ must at least equal $\Delta U$ at the instant that a particle crosses the top of its barrier. As noted before, in the limit $\tau=1$ an amount $c x_{0}^{2}$ of energy oscillates between potential and spring energy for a (nearly) stationary soliton. The energies calculated numerically for the two extreme points in the cycle for $\tau=\Omega=0.8$ are presented in Table I, together with their time-averaged values and their magnitudes calculated from the continuum-limit expressions. The extreme points in the cycle are those illustrated in Figs. 1 and 2 for solitons with $\tau=\Omega=0.8$.

TABLE I. Comparison of numerically calculated dimensionless energy, barrier height, and momentum values for soliton with $\tau=\Omega=0.8$ (far from the continuum limit) with corresponding values calculated from exact and continuum-limit formulas. Here $E_{k}, E_{p}, E_{s}$, and $E$ refer to kinetic energy, potential energy relative to minima of double-minimum wells, potential energy stored in springs, and total energy, respectively. The "odd" configuration is shown for this $\tau=\Omega=0.8$ soliton on potential $k$ of Fig.4. It is called "odd" because with a particle in one well at the well center $(y=0)$, there is an odd number of particles between any two particles with equal and opposite dis-

\begin{tabular}{|c|c|c|c|c|c|}
\hline \multirow{3}{*}{ Parameter $^{\mathrm{a}}$} & \multicolumn{4}{|c|}{ Discrete-case value } & \multirow{3}{*}{$\begin{array}{c}\text { Continuum } \\
\text { formula } \\
\text { value }\end{array}$} \\
\hline & \multicolumn{2}{|c|}{ Configuration } & \multicolumn{2}{|c|}{ Average value } & \\
\hline & Odd & Even & $\frac{1}{2}($ Odd + Even $)$ & Time & \\
\hline$E_{k}$ & 0.40445 & 0.37232 & 0.38838 & & 837 \\
\hline$E_{p}$ & 0.18829 & $\underline{0.20402}$ & $\underline{0.19616}$ & $\underline{0.19616}$ & $\underline{0.34405}$ \\
\hline$E_{k}+E_{p}$ & 0.59274 & 0.57634 & 0.58454 & 0.58453 & 0.73242 \\
\hline${ }^{n} E_{s}$ & 0.67132 & 0.68772 & $0.67952^{\circ}$ & 0.67953 & 0.73242 \\
\hline$E$ & 1.26406 & 1.26406 & 1.26406 & $1.26406^{b}$ & 1.46484 \\
\hline$U(0)-U\left(x_{0}\right)$ & $\cdots$ & $\ldots$ & & $0.10532^{b}$ & 0.28348 \\
\hline$p$ & 1.46296 & 1.44983 & 1.45640 & & 637 \\
\hline
\end{tabular}
placements. The middle chains in Figs. 1 and 2 have the odd configuration, while the upper and lower chains have even configurations.

aEnergies in units of $c x_{0}^{2}$, momenta in units of $\left(m c x_{0}^{2}\right)^{1 / 2}$.

bIndependent of time; not a time average. 


\section{DISCUSSION}

In this model the solitons propagate without energy loss because there is no loss mechanism. In a more realistic model there would be at least three mechanisms for energy transfer to and from such solitons. First, phonons in the mass-spring chain are known to exchange energy with solitons in the discrete case although not in the continuum limit. ${ }^{8}$ Second, the potential wells in a physical situation would probably result from interactions with heavy ions, and energy could be exchanged between the solitons and the phonons of the heavy ion system. Third, if an external field or stress lifts the energy degeneracy for the two minima the soliton will gain or lose energy by an amount $U\left(x_{0}\right)-U\left(-x_{0}\right)$ for each lattice space that it moves to the left or right. The soliton would accelerate or decelerate accordingly, by an amount governed by Eq. (35) in the continuum limit.

We have shown in what manner the continuumlimit expressions break down near the short-soliton limit and have obtained closed expressions for the shape of the potential well and for the several contributions to the soliton energy, given the form $x_{n}=x_{0} \tanh (\omega t-k n a)$ for the particle displacement field. We have further shown that for such solitons the maximum energy is $2 c x_{0}^{2}$ and the maximum barrier height is $c x_{0}^{2}$; for higher barriers (relative to the spring energy) particles must propagate by thermally activated hopping rather than by the correlated motions involved in soliton propagation.

From Fig. 6 it appears that the continuum-limit expressions are reasonably accurate for $\tau$ less than about 0.6 . This corresponds to solitons longer than about $3 a$ (three lattice spaces), if soliton length is taken as $2(k a)^{-1}$. In terms of relative barrier height, the continuum approximation is good for $\Delta V<0.2$, the discrete case expressions are required for $0.2 \leqslant \Delta V \leqslant 1$, and solitons cannot exist for $\Delta V>1$. Accordingly there is a range of about a factor of 5 in relative barrier height for which the discrete case expressions are necessary in describing soliton behavior for this model. A similar relation between soliton length and the importance of discreteness effects has been noted by Currie et al. ${ }^{9}$ for systems obeying the sine-Gordon equation.

A limitation of the present model is that each $(\omega, k)$ combination has a different well shape $U(x)$. One would like to be able to choose some $U(x)$, perhaps a double-minimum potential calculated from the atomic configuration in a certain crystal, and then calculate the atomic displacements for solitons of various velocities propagating along a chain of coupled particles residing in such double-minimum wells. A possible extension of our model to achieve this purpose would be generalization of the soliton shape to the form $x_{n}=x_{1} T_{n}+x_{3} T_{n}^{3}+x_{5} T_{n}^{5}+\cdots$, where $T_{n} \equiv \tanh (\omega t-k n a)$ as before. The equation of motion would yield a power series in $T^{2}$ of the form of Eq. (10), and the coefficients $A, B, C, D, \ldots$ in the potential could be found by requiring that the coefficient of each power of $T^{2}$ vanish. The complexity of the calculation would increase rapidly with the number of terms in $x_{n}$, and it may prove impractical to go beyond the $x_{3} T_{n}^{3}$ term. Even with only two terms, quite a range of soliton "shape" is possible; for instance, for certain ranges of $x_{3} / x_{1}$ each particle involved in soliton propagation will overshoot its new potential minimum position and then return and settle down in it.

To employ this extended model to determine how the soliton shape varies with the soliton-velocity parameter $\Omega$ for a given potential, one could vary $\tau$ and the $x_{3} / x_{1}$ ratio for each given value of $\Omega$, and find which $\left(\tau, x_{3} / x_{1}\right)$ combination best fits the desired potential form. As an example of the analogous procedure for the present model, choose a potential $V$ of barrier height 0.02 units and refer to Fig. 3. As $\Omega$ is increased from 0 to $0.6, \tau$ must be increased from 0.2 to about 0.6 to keep the barrier height constant. It is evident from curves $a$ and $g$ that as the soliton velocity increases for approximately the same potential, the soliton becomes shorter (involves fewer particles of significant displacement). The shape of $V(y)$ changes significantly from curve $a$ to curve $g$. The expected advantage of the extended model is that with two parameters ( $\tau$ and $x_{3} / x_{1}$ ) or more to manipulate instead of the single parameter $\tau$, it will possible to keep both the height and the shape of $V(y)$ nearly constant as $\Omega$ is varied.

\section{APPENDIX}

A procedure, due to Kinnersley, ${ }^{16}$ for integrating one of the terms in Eq. (25) to obtain $\left\langle E_{p}\right\rangle$ is presented here. The integral for this term is

$$
F(\tau)=\int_{0}^{1} d y\left(1-y^{2}\right)^{-1} \ln \left(\frac{1-\tau^{2} y^{2}}{1-\tau^{2}}\right) .
$$

We note that

$$
\begin{aligned}
\frac{d F}{d \tau} & =\int_{0}^{1} d y\left(1-y^{2}\right)^{-1}\left[-2 \tau y^{2}\left(1-\tau^{2} y^{2}\right)^{-1}+2 \tau\left(1-\tau^{2}\right)^{-1}\right] \\
& =2 \tau \int_{0}^{1} d y\left(1-y^{2}\right)^{-1}\left(1-\tau^{2} y^{2}-y^{2}+\tau^{2} y^{2}\right) /\left[\left(1-\tau^{2} y^{2}\right)\left(1-\tau^{2}\right)\right] \\
& =2 \tau\left(1-\tau^{2}\right)^{-1} \int_{0}^{1} d y\left(1-\tau^{2} y^{2}\right)^{-1}=2\left(1-\tau^{2}\right)^{-1} \tanh ^{-1} \tau
\end{aligned}
$$


Since $F(0)=0$ and $\tau \equiv \tanh (k a)$,

$$
F(\tau)=\int_{0}^{\tau}\left(\frac{d F}{d \tau}\right) d \tau=\int_{0}^{\tau} 2 d \tau\left(1-\tau^{2}\right)^{-1} \tanh ^{-1} \tau=\left(\tanh ^{-1} \tau\right)^{2}=k^{2} a^{2}
$$

\section{ACKNOWLEDGMENTS}

The author thanks William Kinnersley for helpful discussions and mathematical assistance, and George Tuthill and Steve Torstveit for reading the manuscript. Support from NSF Grant No. DMR78-09205 is gratefully acknowledged.

${ }^{1}$ A. C. Scott, F. Y. F. Chu, and D. W. McLaughlin, Proc. IEEE 61,1443 (1973).

${ }^{2}$ D. W. McLaughlin, J. Math. Phys. 16, 96 (1975).

${ }^{3}$ J. A. Krumhansl and J. R. Schrieffer, Phys. Rev. B 11, 3535 (1975).

${ }^{4}$ G. F. Mazenko and P. S. Sahni, Phys. Rev. B $\underline{18}, 6139$ (1978).

${ }^{5}$ A. R. Bishop, E. Domany, and J. A. Krumhansl, Phys. Rev. B 14, 2966 (1976).

${ }^{6} Y$. Ishibashi and Y. Takagi, J. Phys. Soc. Jpn. 33, 1 (1972).

${ }^{7}$ S. Aubry and R. Pick, Ferroelectrics $\underline{8}, 471$ (1974).

${ }^{8}$ T. R. Koehler, A. R. Bishop, J. A. Krumhansl, and J. R. Schrieffer, Solid State Commun. 17, 1515 (1975).

${ }^{9}$ J. F. Currie, S. E. Trullinger, A. R. Bishop, and J. A. Krumhansl, Phys. Rev. B 15, 5567 (1977).
${ }^{10}$ R. A. Guyer and M. D. Miller, Phys. Rev. A $\underline{17}, 1205$ (1978).

${ }^{11}$ R. A. Guyer and M. D. Miller, Phys. Rev. A 17,1774 (1978).

${ }^{12}$ M. J. Ablowitz and J. F. Ladik, J. Math. Phys. 16,598 (1975).

${ }^{13}$ M. J. Ablowitz and J. F. Ladik, Stud. Appl. Math. $\underline{55}, 213$ (1976).

${ }^{14} \mathrm{~V}$. H. Schmidt, The Hydrogen Bond (North-Holland, New York, 1976), Vol. III, p. 1109; M. E. Lines and A. M. Glass, Principles and Applications of Ferroelectrics and Related Materials (Clarendon, Oxford, 1977).

${ }^{15}$ W. Känzig and R. Sommerhalder, Helv. Phys. Acta 26, 603 (1953).

${ }^{16}$ W. Kinnersley (private communication). 